\title{
The WWOX gene inhibits the growth of U266 multiple myeloma cells by triggering the intrinsic apoptotic pathway
}

\author{
HONGSHENG ZHANG ${ }^{1}$, LINGYING KONG ${ }^{1}$, ZHAOLEI CUI ${ }^{1}$, WEI DU ${ }^{1}$, \\ YIHUI HE ${ }^{2}$, ZHI YANG ${ }^{1}$, LI WANG ${ }^{1}$ and XIAOYAN $\mathrm{CHEN}^{2}$ \\ ${ }^{1}$ Department of Pathology, Affiliated People's Hospital of Fujian University of Traditional Chinese Medicine, Fuzhou, \\ Fujian 350004; ${ }^{2}$ Department of Pathology, Fujian Provincial Hospital, Fuzhou, Fujian 350001, P.R. China
}

Received March 5, 2014; Accepted June 19, 2014

DOI: $10.3892 /$ ijmm.2014.1824

\begin{abstract}
The role of the WW domain-containing oxidoreductase (WWOX) gene in multiple types of solid human cancers has been documented extensively. However, the functional role of WWOX in human multiple myeloma has not yet been fully elucidated. The present study aimed to investigate the effects of exogenous WWOX expression on the biological properties of U266 multiple myeloma cells, as well as the possible molecular mechanisms involved. In vitro experiments revealed that exogenous WWOX cDNA transfection resulted in marked growth arrest and the induction of apoptosis in the U266 multiple myeloma cells, accompanied by the activation of the intrinsic apoptotic pathway. Our data provide evidence that WWOX also plays a role as a tumor suppressor gene in multiple myeloma, possibly by suppressing cell proliferation and promoting apoptosis by triggering the intrinsic apoptotic pathway.
\end{abstract}

\section{Introduction}

The full-length human WW domain-containing oxidoreductase (WWOX) gene which encodes the Wwox protein located at 16q23.3-24.1, a chromosome region that spans one of the most active common fragile sites, termed FRA16D (1). To date, extensive research into the WWOX gene has suggested that WWOX may function as a tumor suppressor gene, as it behaves aberrantly and affects multiple types of cancer, such as breast, lung, bladder, ovarian, liver, esophageal and pancreatic cancer (2-7). Notably, a restoration or upregulation of WWOX expression is capable of promoting apoptosis in vitro and inhibits tumor growth in vivo $(8,9)$. In addition, studies using WWOX knockout mice have demonstrated increased

Correspondence to: Dr Hongsheng Zhang, Department of Pathology, Affiliated People's Hospital of Fujian University of Traditional Chinese Medicine, No. 602, Middle Road 817, Taijiang, Fuzhou, Fujian 350004, P.R. China

E-mail: 550426851@qq.com

Key words: WW domain-containing oxidoreductase, re-expression, U266 multiple myeloma cells, proliferation, apoptosis, mechanism tumor susceptibility, as well as defects in bone and calcium metabolism $(10,11)$. Moreover, a reduced WWOX expression is commonly observed in cancer cases and is associated with a poor prognosis $(12,13)$. Therefore, WWOX is expected to be a potential target for the gene-targeted therapy of human carcinomas.

The aberration or absence of WWOX expression in primary hematopoietic malignancies has also been reported, suggesting that WWOX also plays an important role in human hematopoietic malignancies (14-16). Recent studies have revealed that WWOX exerts a role as an anti-oncogene in leukemia, which inhibits cell proliferation and promotes apoptosis through the mitochondrial pathway $(17,18)$. Furthermore, it has been confirmed that WWOX is a pro-apoptotic protein that is closely related to apoptosis-associated factors, including Bcl-2, Bcl-xL, Bax, caspases, p73 and p53 involved in apoptotic signaling pathways (19-21). As there is no report on the functional role of WWOX in human multiple myeloma, and to confirm the functional role of WWOX in suppressing multiple myeloma cells, in this study, we transfected WWOX cDNA into U266 multiple myeloma cells (Wwox protein-negatvie) utilizing the constructed pCMV-WWOX-EGFP vector. We investigated the effects of Wwox protein re-expression on the biological properties of U266 multiple myeloma cells, as well as the underlying molecular mechanisms. Our data revealed that Wwox re-expression resulted in a significant suppression of cell viability and the induction of apoptosis in U266 human multiple myeloma cells, possibly by activating the intrinsic apoptotic pathway.

\section{Materials and methods}

Materials. The U266 multiple myeloma cell line was purchased from the Chinese Academy of Sciences (Shanghai, China). The main reagents used in this study are listed as follows: RPMI1640 medium and fetal bovine serum (FBS) (Gibco-BRL, Carlsbad, CA, USA); RT-PCR kit (Fermentas, Glen Burnie, MD, USA); TRIzol reagent and pfu DNA polymerase (Thermo Fisher Scientific, Waltham, MA, USA); pCMV-EGFP vector, HindIII, Xhol enzyme, T 4 DNA ligase, RIPA lysis buffer, DAPI, Cy3-conjugated anti-rabbit $\operatorname{IgG}, \mathrm{Cy} 3$-conjugated anti-mouse IgG, mouse anti-human $\beta$-actin, anti-cytochrome $c$, rabbit anticleaved PARP and anti-cleaved caspase-3 (Asp175) (Beyotime 
Institute of Biotechnology, Shanghai, China); rabbit antihuman Wwox, anti-Bcl-2 and anti-Bax (Abcam, Cambridge, MA, USA); rabbit anti-caspase-9 and anti-caspase-3 (Santa Cruz Biotechnology, Santa Cruz, CA, USA); GenFectin ${ }^{\mathrm{TM}}$ Transfection kit, Plasmid Extraction Mini kit and DNA purification kit (Beijing Zoman Biotechnology, Beijing, China); Cell Counting Kit-8 (Dojindo Laboratories, Kumamoto, Japan).

Cell culture. U266 human myeloma cells were maintained in RPMI-1640 medium supplemented with $10 \%$ fetal bovine serum (FBS), and cultured at $37^{\circ} \mathrm{C}$ in $5 \% \mathrm{CO}_{2}$.

Vector construction. Total RNA was extracted using TRIzol reagent from normal human peripheral blood mononuclear cells and was reverse transcribed into cDNA using a commercial RT-PCR kit. The full-length WWOX cDNA was cloned with the primer pairs (contains HindIII and Xhol enzyme cutting sites which are shown by the underlined letters): forward, 5'-CCGAAGCTTGCACCATGGCAGCGCTGCGC-3' and reverse, 5'-CCGCTCGAGTTAGCCGGACTGGC-3'. The PCR products (1268 bp) and pCMV-EGFP vector were all digested by both HindIII and Xhol enzymes followed by purification using a commercial DNA purification kit. The digested WWOX cDNA and pCMV-EGFP were integrated with the aid of the $\mathrm{T}_{4}$ DNA ligase, followed by transformation, clone picking and amplification. The combined plasmids were extracted using a Plasmid Extraction Mini kit and sent for sequencing.

Cell transfection and cell growth assays. In this section, pCMV-WWOX-EGFP (encoding Wwox-GFP fusion protein), pCMV-EGFP (a mock plasmid only encoding GFP) and nontreated groups (blank control) were established. The cells were seeded at a density of $1 \times 10^{5} / \mathrm{ml}$, and transfected using the GenFectin ${ }^{\mathrm{TM}}$ Transfection kit according to the specified protocol. A commercial Cell Counting Kit- 8 was used to evaluate the growth inhibitory effects following the manufacturer's instructions. The optical density (OD value proportional to the cell number) was measured using a microculture plate reader (BioTek Instruments, Winooski, VT, USA) at both 450 and $630 \mathrm{~nm}$.

Colony-forming assay. Briefly, GFP-expressing cells were sorted by a special sterile flow cytometry using Vi-Cell counter (Beckman Coulter, Inc., Fullerton, CA, USA), and 300 cells/ml were seeded in 24-well plates. Methylcellulose dissolved in RPMI-1640 containing 30\% FBS was supplied to each well at a concentration of $0.8 \mathrm{~g} / \mathrm{l}$. The colonies containing $>50$ cells were counted following incubation at $37^{\circ} \mathrm{C}$ in $5 \% \mathrm{CO}_{2}$ for 2 weeks.

Analysis of apoptosis. DAPI fluorescence staining and DNA fragmentation were employed in the assessment of cell apoptosis following transfection. For the DAPI fluorescence staining, the cells were harvested, washed with PBS and stained with DAPI ( $2 \mathrm{mg} / \mathrm{ml})$ for 3-5 min followed by washing 2 times with PBS. The morphological changes of the stained cells were observed using a fluorescence microscope (Olympus, Tokyo, Japan) at x400 magnification. Non-viable apoptotic ratio (\%) = (cell numbers in phase IIb/200 cells) x100\%. DNA fragmentation analysis was performed according to the protocols of a commercial DNA Fragmentation kit (Beyotime Institute of Biotechnology), followed by $1.0 \%$ agarose gel electrophoresis with a constant voltage of $18 \mathrm{~V}$ for $>4 \mathrm{~h}$.

Immunofluorescence. Beforehand, the GFP-expressing cells were sorted by flow cytometry using a specified sterile Vi-Cell counter (Beckman Coulter). The cell monolayer was fixed with $4 \%$ paraformaldehyde, and the cells were incubated at $4^{\circ} \mathrm{C}$ overnight with rabbit anti-cleaved caspase-3 (Asp175) (1:300) and mouse monoclonal anti-cytochrome $c$ (1:300) antibodies. Cy3-conjugated anti-rabbit and anti-mouse IgG were all diluted at 1:1,000. DAPI was used to dye the cell nuclei. The stained cells were washed with PBS and observed with a fluorescence microscope (Olympus) at x400 magnification.

Real-time PCR. Real-time PCR was performed using a SYBR-Green PCR Master Mix (Roche Diagnostics GmbH, Mannheim, Germany) under the recommended conditions. The primer sequences for Bcl-2, Bax and GAPDH were previously described (17). The comparative $\mathrm{Ct}$ method to GAPDH was employed to calculate the mRNA expression level of Bcl-2 and Bax.

Immunoblotting. Cell lysates were prepared using RIPA protein lysis buffer and the protein extracts were quantified and then subjected to electrophoresis on a 10-12\% SDS-PAGE gel. The proteins were transferred onto polyvinylidene difluoride (PVDF) membranes and blocked in Tris-buffered saline (TBS) containing 5\% non-fat milk powder. The primary antibodies and the dilutions used were: rabbit anti-Wwox $(1: 1,000)$, anti-Bcl-2 (1:1,000), anti-caspase-9 (1:500), anti-caspase-3 (1:500) and anti-cleaved PARP (1:300). Mouse monoclonal anti-cytochrome $c$ antibody was diluted at 1:500, while mouse monoclonal anti- $\beta$-actin antibody was diluted at 1:1,000 and used as a positive control.

Statistical analysis. Data are presented as the means \pm standard deviation (SD). One-way ANOVA, the Student's t-test or the non-parametric test were used for comparison differences between groups using SPSS 13.0 software (SPSS Inc., Chicago, IL, USA), and statistical significance for the data was set at $\mathrm{P}$-value $<0.05$.

\section{Results}

Exogenous WWOX cDNA was successfully cloned and effectively transfected into the U266 cells. We first examined whether the pCMV-WWOX-EGFP vector was correctly recombinated: the DNA sequencing results exhibited that the full length WWOX cDNA was correctly connected to the plasmid (Fig. 1A). We further evaluated whether Wwox protein was successfully restored or re-expressed in the U266 cells using a fluorescence microscope and immunoblotting. The results indicated that the pCMV-WWOX-EGFP- and pCMV-EGFP-transfected cells all expressed GFP at $36 \mathrm{~h}$ following transfection (Fig. 1B). In addition, the re-expression of Wwox protein was further confirmed by immunoblotting using rabbit anti-human Wwox antibody: an expected 74-kDa Wwox-GFP fusion protein at $72 \mathrm{~h}$ following transfection was detected (Fig. 1C). 
A

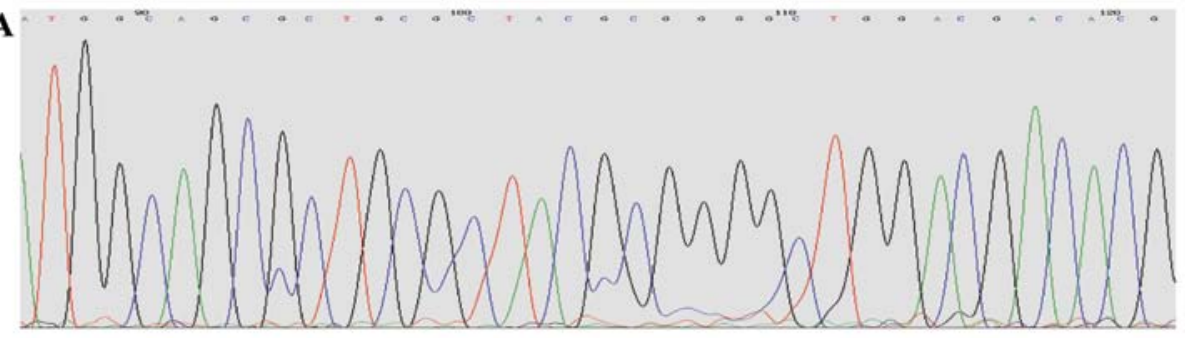

B

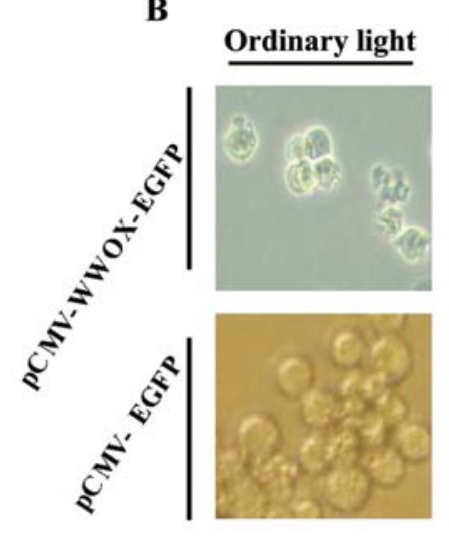

C
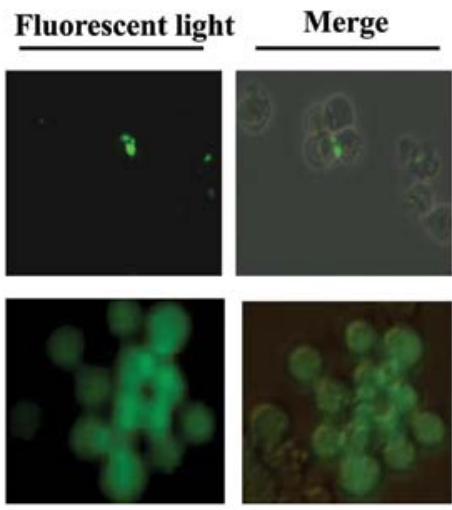
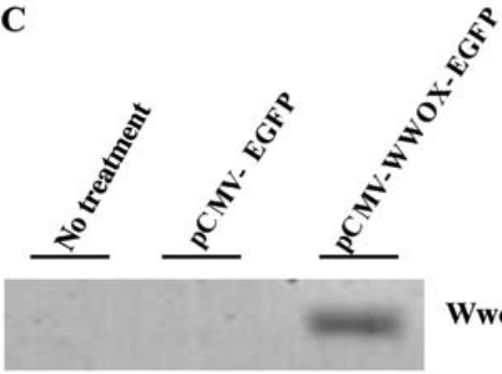

Wwox-GFP

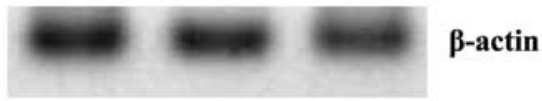

$\beta$-actin

Figure 1. DNA sequencing of the recombined vector and expression of Wwox-GFP in U266 multiple myeloma cells following transfected with the vectors. (A) DNA sequencing of the recombined vector, pCMV-WWOX-EGFP. (B) Expression of Wwox-GFP fusion protein and mock vector GFP were observed at $36 \mathrm{~h}$ after transfection using a fluorescence microscope, at x200 magnification. (C) Expression of Wwox-GFP fusion protein at $72 \mathrm{~h}$ after transfection detected by immunoblotting.

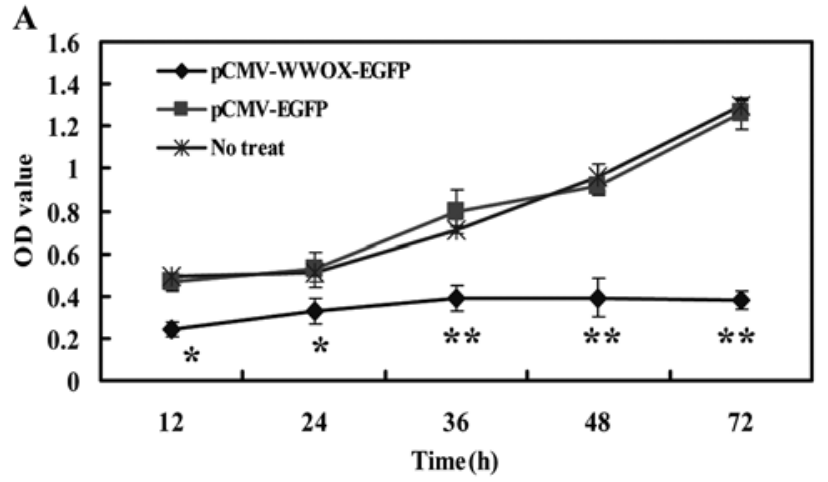

B

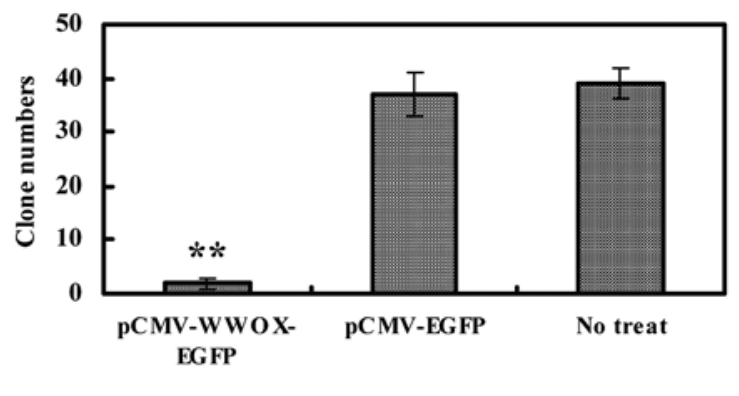

Figure 2. Effects of Wwox re-expression on the cell viability and colony formation of U266 multiple myeloma cells. (A) Cell Counting Kit-8 assay was used to evaluated the inhibitory effects of Wwox re-expression on cell viability. The OD value proportional to the cell number was measured and plotted on the growth curve. (B) Colony-forming assay. Cell colonies containing $>50$ cells were counted on day 14 . Data shown are the means $\pm S D(n=15)\left({ }^{*} \mathrm{P}<0.05 ;{ }^{* *} \mathrm{P}<0.01\right.$ vs. untreated cells).

Re-expression Wwox reduces cell viability and colony formation. The effects of Wwox protein re-expression or restoration on the viability of U266 cells were assessed by CCK-8 assay. The results revealed that pCMV-WWOX-EGFP transfection led to a detection of $0.242 \pm 0.037,0.326 \pm 0.059$, $0.386 \pm 0.061,0.393 \pm 0.089$ and $0.379 \pm 0.042$ of the OD values (proportional to the cell numbers) at $12,24,36,48$ and $72 \mathrm{~h}$ following transfection, respectively; significantly lower than those of non-treated cells with $\mathrm{P}<0.05$ or 0.01 . By contrast, pCMV-EGFP mock vector transfection revealed no difference with $\mathrm{P}>0.05$ when compared with the non-treated U266 cells (Fig. 2A). For the colony formation assay, significantly fewer and smaller colonies were formed for the pCMV-WWOXEGFP-transfected cells compared with the untreated cells, while the pCMV-EGFP-transfected cells showed no statistical significance vs. the untreated cells with $\mathrm{P}>0.05$ (Fig. 2B).

pCMV-WWOX-EGFP transfection promotes cell apoptosis. The apoptosis-inducing effects were measured with the aid of DAPI staining and DNA ladder electrophoresis. As displayed in Fig. 3A, DAPI staining exhibited apparent microscopic changes in the nucleis in the pCMV-WWOX-EGFP-transfected U266 cells characterized by a morphology of chromatin condensation and shrinkage in phase IIb of the apoptotic phase at $72 \mathrm{~h}$ 

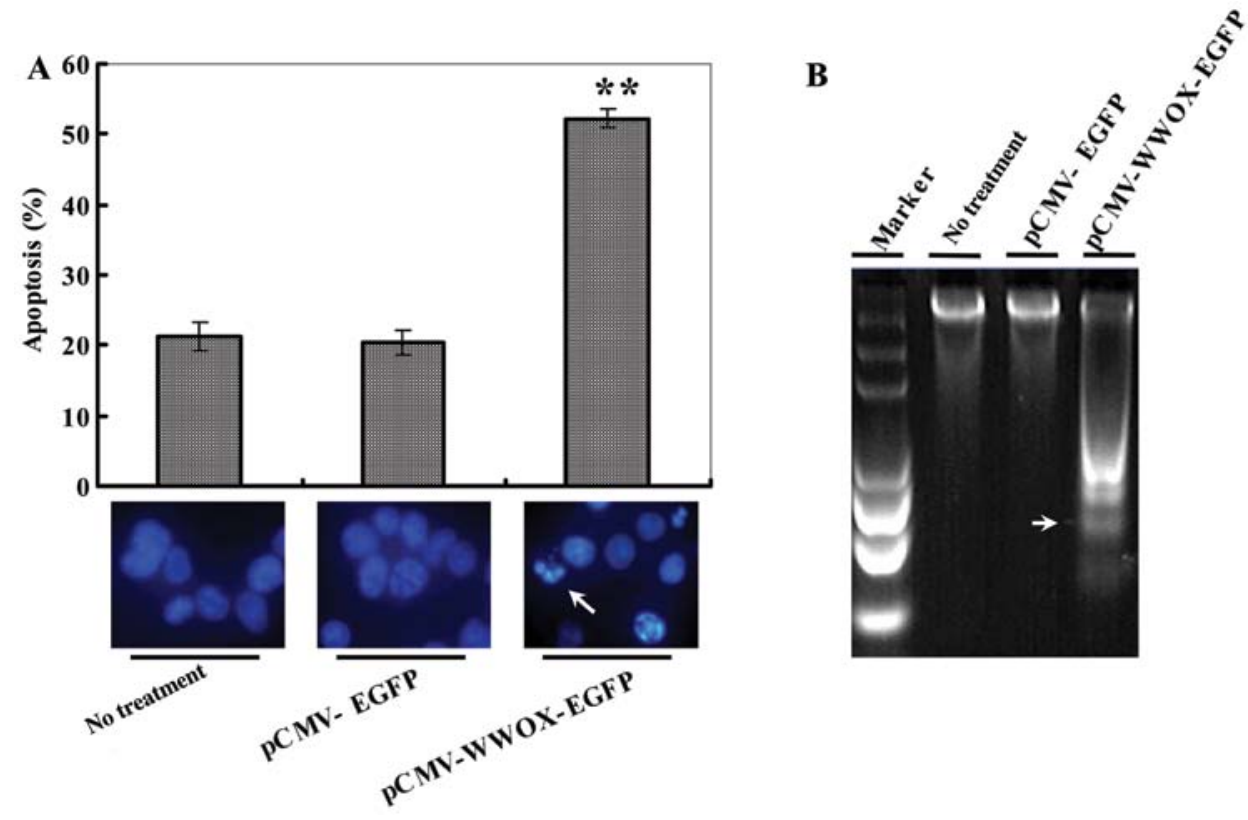

Figure 3. Wwox re-expression promotes apoptosis in U266 multiple myeloma cells. (A) DAPI fluorescence staining shows the microscopic changes in the nuclei of pCMV-WWOX-EGFP-transfected cells observed using a fluorescence microscope, at 400 magnification. The white arrow indicates typical morphology of the nucleus following the apoptosis of the transfected cells at $72 \mathrm{~h}$ following transfection. Non-viable apoptotic ratio (\%) $=($ phase IIb cell numbers/200 cells) $\mathrm{x} 100 \%$. Data shown are the means $\pm \mathrm{SD}(\mathrm{n}=3)\left({ }^{* *} \mathrm{P}<0.01 \mathrm{vs}\right.$. untreated cells). (B) DNA fragmentation analysis. DNA was extracted at $72 \mathrm{~h}$ following transfection and the white arrow shows the typical 'DNA ladders' in pCMV-WWOX-EGFP-transfected cells.

following transfection, while the 2 contrast groups presented no marked apoptotic morphology. Similarly, the non-viable apoptotic ratio (\%) for pCMV-WWOX-EGFP at $72 \mathrm{~h}$ after transfection was $(52.25 \pm 1.38 \%)$, significantly higher than that of the 2 control groups with $\mathrm{P}<0.01$ (Fig. 3A). DNA degradative fragments were detected by DNA ladder electrophoresis, which also presented typical apoptosis 'DNA ladders' in the pCMV-WWOX-EGFP-transfected cells when compared with the controls as shown in Fig. 3B.

The intrinsic apoptotic pathway is involved in the WWOX-mediated induction of apoptosis. Real-time PCR, immunoblotting and immunofluorescence were performed for the detection of apoptosis-related proteins, such as Bcl-2, Bax, PARP, cytochrome $c$, caspase-3 and caspase-9. Real-time PCR showed that the mRNA expressio of Bcl-2 in the pCMVWWOX-EGFP-transfected cells decreased with $\mathrm{P}<0.01$ vs. the pCMV-EGFP-transfected cells, while the Bax mRNA expression changed unconspicuously (Fig. 4A). As shown in Fig. 4B, immunofluorescence revealed that caspase- 3 was activated as well, accompanied by an increase in the levels of cytochrome $c$. Enhanced red fluorescence was observed in the pCMV-WWOXEGFP-transfected cells, utilizing anti-cleaved caspase-3 (Asp175) and anti-cytochrome $c$ antibodies, recognized by Cy3-conjugated anti-mouse or rabbit IgG. Immunoblotting also displayed that the expression of $\mathrm{Bcl}-2$ protein in the pCMV-WWOXEGFP-transfected U266 cells decreased, while cytochrome $c$ expression increased, all with $\mathrm{P}<0.05$ when comparing the pCMV-EGFP-transfected cells with the untreated U266 cells. Both caspase- 3 and -9 were activated, as indicated by presenting their spliceosomes: a $17 \mathrm{kDa}$ spliceosome for pro-caspase-3 and a $37 \mathrm{kDa}$ spliceosome for pro-caspase-9, both observed in the pCMV-WWOX-EGFP-transfected U266 cells. Moreover, cleaved PARP was also detected in the pCMV-WWOX-EGFPtransfected U266 cells (Fig. 4B).

\section{Discussion}

In the present study, we tranfected exogenous WWOX cDNA into U266 human multiple myeloma cells (Wwox proteinnegative) using the pCMV-WWOX-EGFP combination vector and investigated the effects of Wwox re-expression on the biological properties of U266 multiple myeloma cells. Our data exhibited that Wwox re-expression or restoration resulted in a significant suppression of cell viability and the induction of apoptosis in the U266 multiple myeloma cells. We further investigated whether Wwox re-expression activates apoptosisrelated factors involved in the intrinsic apoptotic signaling pathway. As expected, we found that re-expressed Wwox protein downregulated Bcl-2, upregulated cytochrome $c$ and activated PARP, caspase-3 and caspase-9, indicating that Wwox plays a role as a tumor suppressor in U266 cells by activating the intrinsic apoptotic pathway.

Multiple myeloma is considered the second most common hematological malignancy and is characterized by the clonal proliferation of neoplastic plasma cells in the bone marrow. The improved understanding of the molecular mechanisms of multiple myeloma development may provide a basis for the development of effective treatment strategies. The WWOX gene is located at the chromosomal area 16q23.3-24.1, which spans as the common chromosomal fragile site, FRA16D (1). The abnormal expression of WWOX, including high frequency of loss of heterozygosity ( $\mathrm{LOH}$ ), aberrant WWOX mRNA transcripts and reduced expression have been verified in a variety of solid carcinomas $(2-5,22)$, while the restoration or overexpression of WWOX in such cancer cells can sensitize them to apoptosis, 
A

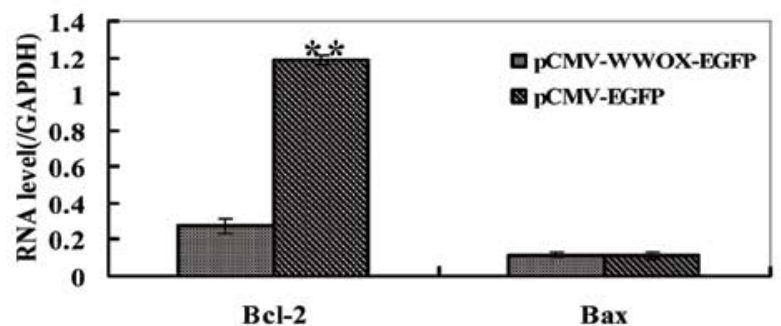

B

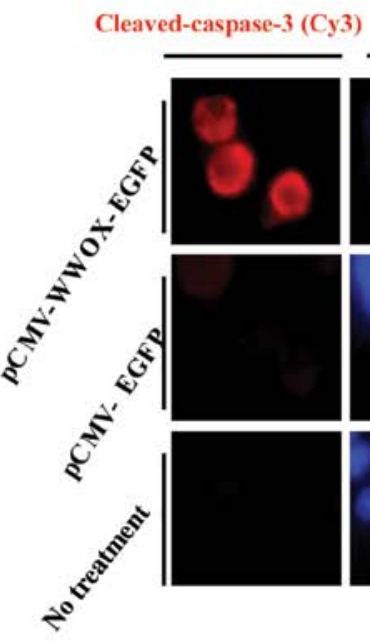

DAPI Merge
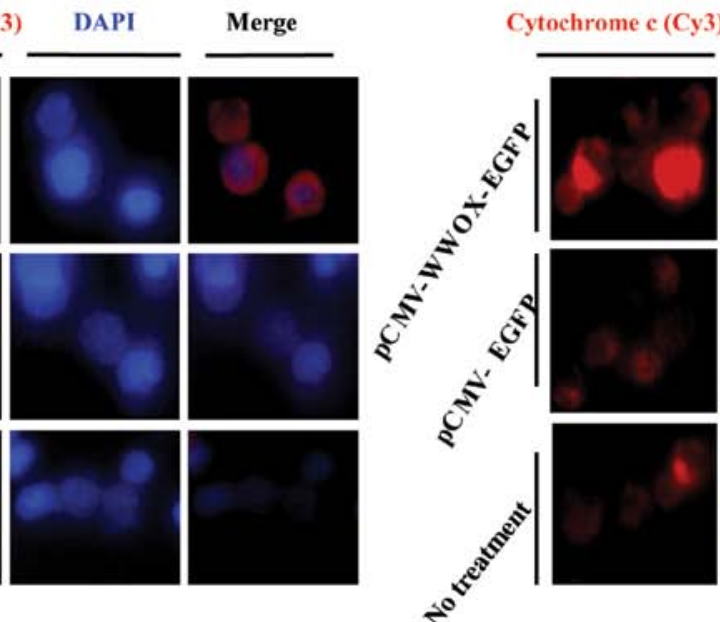

DAPI
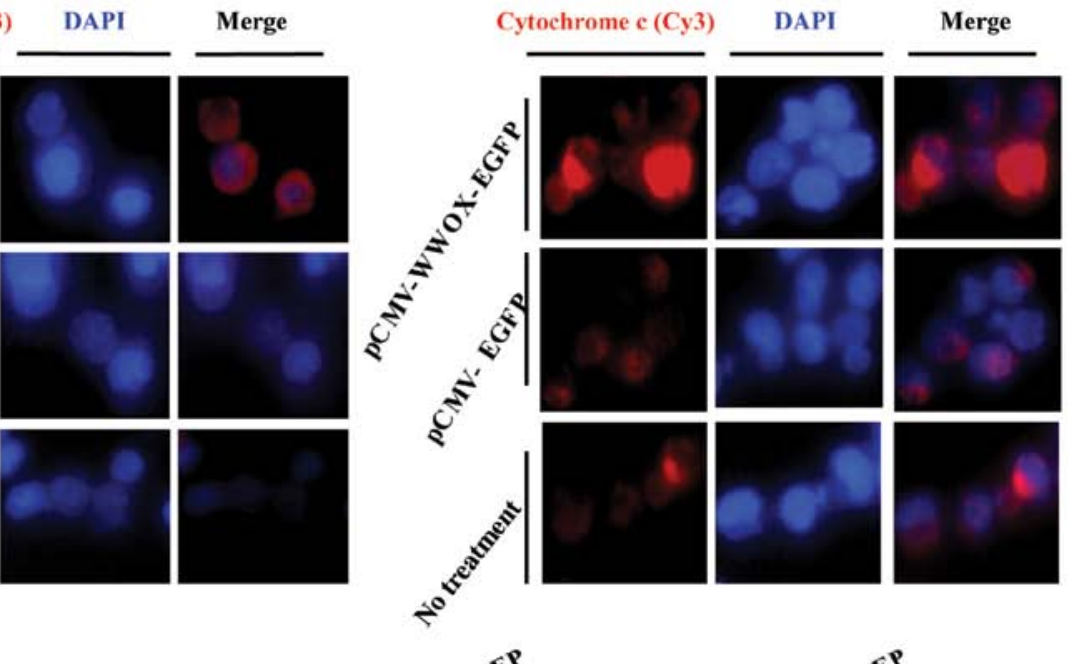

Bcl-2
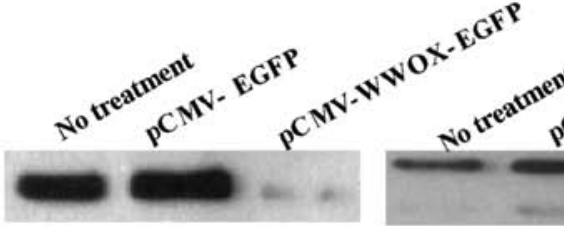

Bax

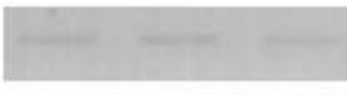

Cytochrome c
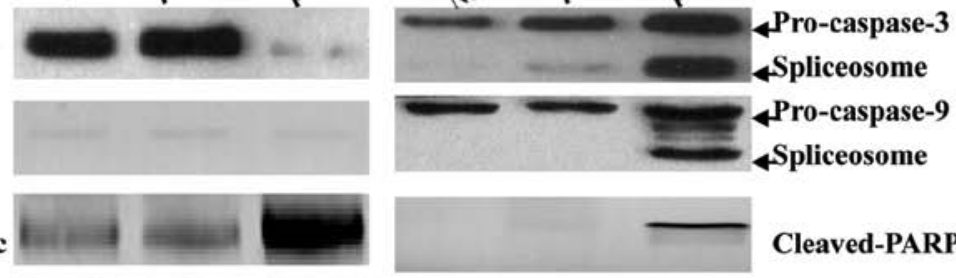

$\beta$-actin

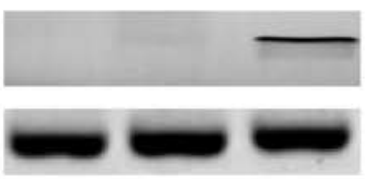

Cleaved-PARP

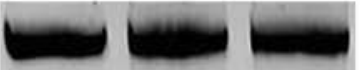

$\beta$-actin

D

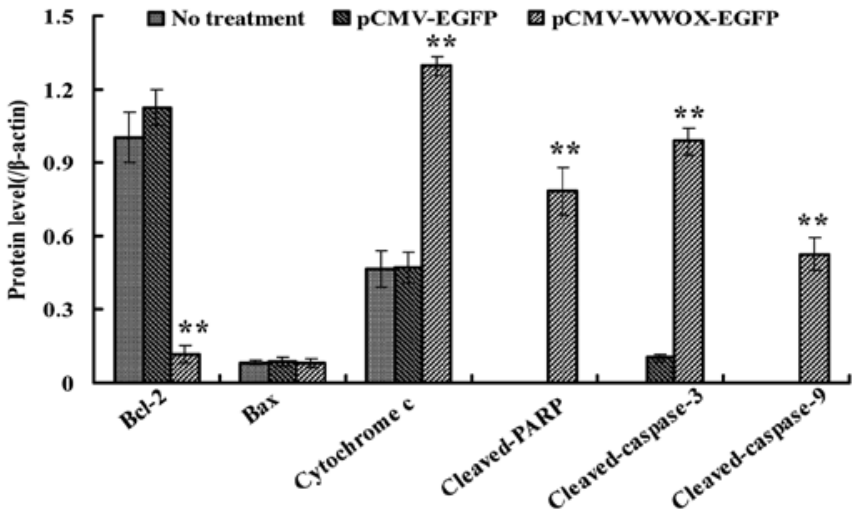

indicating that the WWOX gene plays a role in carcinogenesis and possesses tumor suppressor characteristics $(6,8-10,17)$.

The aberration or absence of WWOX expression in primary hematopoietical malignancies has also been reported (14-18).
Figure 4. Re-expression of Wwox triggers the intrinsic apoptotic pathway. (A) Real-time PCR. Total RNA was extracted at $60 \mathrm{~h}$ following transfection, and the relative quantity was normalized to GAPDH. (B) Immunofluorescence staining, observed using a fluorescence microscope at $60 \mathrm{~h}$ after infection, at $\mathrm{x} 400$ magnification. GFP-expressing cells were sorted by flow cytometry using Vi-Cell counter beforehand. A rabbit anti-human cleaved caspase-3 antibody with a recognition site at Asp175 was used, marked by red fluorescence Cy3-conjugated anti-rabbit IgG. Cytochrome $\mathrm{c}$ was marked by red fluorescence $\mathrm{Cy} 3$-conjugated anti-mouse $\mathrm{IgG}$. The nucleus was stained by DAPI. (C) Immunoblotting analysis of apoptosis-related proteins involved in the intrinsic apoptotic pathway at $72 \mathrm{~h}$ after transfection. (D) Changes in expression of apoptosis-related proteins normalized to $\beta$-actin. Data shown are the means $\pm \mathrm{SD}(\mathrm{n}=3$ or 5$)\left({ }^{* *} \mathrm{P}<0.01\right.$ vs. untreated cells).

Our findings are consistent with those findings: we observed that the re-expression of Wwox protein in the Wwox-negative U266 myeloma cells led to a marked inhibition of cell growth and colony formation, and the apoptotic effects were exhibited 
by the microscopic changes in the nucleus in phase IIb of apoptosis at $72 \mathrm{~h}$ after transfection, accompanied by a higher non-viable apoptotic ratio. Furthermore, typical apoptotic DNA ladders were observed in the pCMV-WWOX-EGFPtransfected U266 cells, suggesting that WWOX promoted the apoptosis of U266 multiple myeloma cells in vitro. Nevertheless, there are opposing viewpoints as to the function role of WWOX as a tumor suppressor gene (23); in contrary with these opposing views, we have confirmed the functional concept that the re-expression of Wwox protein leads to apoptosis in U266 multiple myeloma cells.

WWOX is closely related to apoptosis-associated factors, including Bcl-2, Bcl-xL, Bax, p73 and caspases in its antitumor activities (17-21), indicating that WWOX may play a role in triggering apoptosis-associated pathways. Previous studies have demonstrated that WWOX overexpression results in a downregulation of $\mathrm{Bcl}-2$, as well as a cleavage of both pro-caspase-3 and $-9(19,20)$. It has also been shown that the upregulation of WWOX in cancer cell lines enhances apoptosis by activating the intrinsic apoptotic caspase cascade $(8,9)$. Cui et al (17) recently presented similar results. The present study showed that the ectopic expression of WWOX cDNA resulted in changes in the expression of Bcl-2, cytochrome $c$, PARP, pro-caspase-3 and pro-caspase-9, characterized by a lower expression of Bcl-2 and the increased expression of cytochrome $c$, although Bax expression was not altered significantly. The activation of caspase-3 and -9 was further verified by immunoblotting and immunofluorescence. Cleaved-PARP was also detected, which further provides evidence of the activation of caspase- 3 and -9 . Taken together, it these data suggest that WWOX induces cell death by triggering the intrinsic apoptotic pathway in multiple myeloma. Yet, our findings are supported in part and fully by those of previous studies $(8,9,17-21)$.

In conclusion, the present study reveals a functional role of WWOX in U266 human multiple myeloma cells, as well as the primary molecular mechanisms responsible for its pro-apoptotic effects. However, in the present study we did not evaluate the functional role of WWOX in multiple myeloma in vivo and the clinical characterization of WWOX in multiple myeloma patients. As regards the analysis of apoptosis, we failed to evaluate the apoptotic ratio by flow cytometry marked with PE/7-AAD fluorescent dye, due to the non-detectable cell fragments that may affect by Wwox re-expression. The release of cytochrome $c$ from the mitochondria should also be verified. The mechanisms underlying the molecular action of WWOX in multiple myeloma warrant further investigation and more detailed analyses need to be performed in future studies.

\section{Acknowledgements}

The authors thank Professor Donghong Lin for providing editorial assistance.

\section{References}

1. Bednarek AK, Laflin KJ, Daniel RL, Liao Q, Hawkins KA and Aldaz CM: WWOX, a novel WW domain-containing protein mapping to human chromosome 16q23.3-24.1, a region frequently affected in breast cancer. Cancer Res 60: 2140-2145, 2000 .
2. Iliopoulos D, Guler G, Han SY, et al: Fragile genes as biomarkers: epigenetic control of WWOX and FHIT in lung, breast and bladder cancer. Oncogene 24: 1625-1633, 2005.

3. Lan C, Chenggang W, Yulan B, Xiaohui D, Junhui Z and Xiao W: Aberrant expression of WWOX protein in epithelial ovarian cancer: a clinicopathologic and immunohistochemical study. Int J Gynecol Pathol 31: 125-132, 2012.

4. Ekizoglu S, Muslumanoglu M, Dalay N and Buyru N: Genetic alterations of the WWOX gene in breast cancer. Med Oncol 29: 1529-1535, 2012

5. Guo W, Wang G, Dong Y, Guo Y, Kuang G and Dong Z: Decreased expression of WWOX in the development of esophageal squamous cell carcinoma. Mol Carcinog 52: 265-274, 2013.

6. Hu BS, Tan JW, Zhu GH, Wang DF, Zhou X and Sun ZQ: WWOX induces apoptosis and inhibits proliferation of human hepatoma cell line SMMC-7721. World J Gastroenterol 18: 3020-3026, 2012.

7. Nakayama S, Semba S, Maeda N, Matsushita M, Kuroda Y and Yokozaki H: Hypermethylation-mediated reduction of WWOX expression in intraductal papillary mucinous neoplasms of the pancreas. Br J Cancer 100: 1438-1443, 2009.

8. Fabbri M, Iliopoulos D, Trapasso F, et al: WWOX gene restoration prevents lung cancer growth in vitro and in vivo. Proc Natl Acad Sci USA 102: 15611-15616, 2005.

9. Iliopoulos D, Fabbri M, Druck T, Qin HR, Han SY and Huebner K: Inhibition of breast cancer cell growth in vitro and in vivo: effect of restoration of Wwox expression. Clin Cancer Res 13: 268-274, 2007.

10. Aqeilan RI, Trapasso F, Hussain S, et al: Targeted deletion of Wwox reveals a tumor suppressor function. Proc Natl Acad Sci USA 104: 3949-3954, 2007.

11. Aqeilan RI, Hassan MQ, de Bruin A, et al: The WWOX tumor suppressor is essential for postnatal survival and normal bone metabolism. J Biol Chem 283: 21629-21639, 2008.

12. Göthlin Eremo A, Wegman P, Stål O, Nordenskjöld B, Fornander $\mathrm{T}$ and Wingren $\mathrm{S}$ : Wwox expression may predict benefit from adjuvant tamoxifen in randomized breast cancer patients. Oncol Rep 29: 1467-1474, 2013.

13. Cancemi L, Romei C, Bertocchi S, et al: Evidences that the polymorphism Pro-282-Ala within the tumor suppressor gene WWOX is a new risk factor for differentiated thyroid carcinoma. Int J Cancer 129: 2816-2824, 2011.

14. Ishii H, Vecchione A, Furukawa Y, et al: Expression of FRA16D/WWOX and FRA3B/FHIT genes in hematopoietic malignancies. Mol Cancer Res 1: 940-947, 2003.

15. Ishii $\mathrm{H}$ and Furukawa $\mathrm{Y}$ : Alterations of common chromosome fragile sites in hematopoietic malignancies. Int J Hematol 79: 238-242, 2004.

16. Chen X, Zhang H, Li P, Yang Z, Qin L and Mo W: Gene expression of WWOX, FHIT and p73 in acute lymphoblastic leukemia. Oncol Lett 6: 963-969, 2013.

17. Cui Z, Lin D, Cheng F, et al: The role of the WWOX gene in leukemia and its mechanisms of action. Oncol Rep 29: 2154-2162, 2013.

18. Lin D, Cui Z, Kong L, Cheng F, Xu J and Lan F: p73 participates in WWOX-mediated apoptosis in leukemia cells. Int J Mol Med 31: 849-854, 2013.

19. Chang NS: A potential role of p53 and WOX1 in mitochondrial apoptosis (Review). Int J Mol Med 9: 19-24, 2002.

20. Yang JL and Zhang W: WWOX tumor suppressor gene. Histol Histopathol 23: 877-882, 2008.

21. Zhang P, Jia R, Ying L, Liu B, Qian G, Fan X and Ge S: WWOX-mediated apoptosis in A549 cells mainly involves the mitochondrial pathway. Mol Med Rep 6: 121-124, 2012.

22. Płuciennik E, Kośla K, Wójcik-Krowiranda K, Bieńkiewicz A and Bednarek AK: The WWOX tumor suppressor gene in endometrial adenocarcinoma. Int J Mol Med 32: 1458-1464, 2013.

23. Watanabe A, Hippo Y, Taniguchi H, et al: An opposing view on WWOX protein function as a tumor suppressor. Cancer Res 63: 8629-8633, 2003. 